\title{
Perspectives of Carbon Paste Electrodes in Stripping Potentiometry
}

\author{
Ivan S̆vancara, * Božidar Ogorevc, ${ }^{* *}$ Samo B. Hočevar, $* *$ and Karel VyTřas ${ }^{* \dagger}$ \\ *Department of Analytical Chemistry, Faculty of Chemical Technology, University of Pardubice, \\ nám. Čs. legií 565, CZ-53210 Pardubice, Czech Republic \\ **Analytical Chemistry Laboratory, National Institute of Chemistry, Hajdrihova 19, P.O. Box 3430, \\ SI-1001 Ljubljana, Slovenia
}

\begin{abstract}
The testing of various types of carbon paste electrodes (CPEs) for their use in stripping potentiometry was carried out by means of specially proposed procedures and by using various model analyte systems. CPEs containing three different pasting liquids (paraffin oil, silicone oil, tricresyl phosphate) were tested as supports for mercury- and gold films, and as substrates for electrolytic, adsorptive, ion-pair forming, and extractive accumulation. Test measurements in organic solvent-containing media and studies of some irreversible electrochemical reactions were performed as well. Individual examples to be studied allowed one to formulate and outline some perspectives of CPEs in potentiometric stripping analysis and related constant current stripping analysis.
\end{abstract}

(Received October 15, 2001; Accepted December 19, 2001)

\section{Introduction}

Potentiometric stripping analysis (PSA) and related constant current stripping analysis (CCSA) have been first introduced by Jagner and his group ${ }^{1}$ as modified versions of traditional chronopotentiometry. ${ }^{2}$ Due to an effective combination of classical chronopotentiometric principles with a preconcentration step and with powerful computerized instrumentation, both PSA and CCSA belong now among the most effective electrochemical techniques, meeting almost all criteria required for modern analysis..$^{3-5}$ They are relatively insensitive to matrix effects and are capable of operating at extremely low concentration levels. In addition, the measurements are typically unaffected by the presence of aerial oxygen in the sample, which further simplifies the analytical procedures employing either PSA or CCSA. Possibilities of stripping potentiometric methods in practical electroanalysis are also closely connected with the choice of a suitable working electrode.

Carbon paste electrodes (CPEs) as sensors commonly used in stripping voltammetry ${ }^{6,7}$ are, in principle, applicable in similar PSA or CCSA. However, compared to extensive voltammetric applications, CPEs have been combined with stripping potentiometry only occasionally ${ }^{8,9}$ and some valuable properties of these electrodes remained practically unnoticed. Among them, one can quote very favorable signal-to-noise characteristics of CPEs within a wide anodic potential range utilisable mainly for oxidations of organic compounds or unique interactions between the carbon paste and an analyte. Here, either a binder or a suitable modifier may participate in various processes at the electrode, such as extraction, ion-pair formation or catalyzed reactions. ${ }^{10}$ Carbon paste can be modified with

\footnotetext{
† To whom correspondence should be addressed.

E-mail: karel.vytras@upce.cz
}

chemical or biological agents and usually more simply compared to modifications of solid electrode substrates. ${ }^{6,7}$ As confirmed recently, ${ }^{11-13}$ e.g. extraction and ion-pairing processes at CPEs originally designed for voltammetric experiments ${ }^{14,15}$ are highly effective even in CCSA. Some other processes and types of measurements with CPEs mentioned above are also potentially exploitable in stripping potentiometry. ${ }^{4,5}$

That the use of carbon paste-based electrodes and their modified variants may significantly extend applications of both PSA and CCSP is shown in this article. The results and observations that are summarised in the following sections represent more or less initial studies on the use of several types of CPEs in stripping potentiometry by using various model systems and with the aid of different methodical principles.

\section{Experimental}

\section{Apparatus}

An AUTOLAB PGSTAT 10 system equipped with ECDMODUL and controlled by GPES software (Ecochemie, Utrecht, Holland) was used in combination with an electrode stand (Metrohm, Switzerland) adapted for measurements with CPEs. Besides a CPE (see below), the electrode cell involved also a Pt-wire auxiliary and an $\mathrm{Ag} / \mathrm{AgCl}$ reference electrode (saturated solution of $\mathrm{KCl}$; Metrohm, Switzerland). Stirring was performed with a Teflon-coated bar at approx. $100 \mathrm{rpm}$ using a magnetic stirrer (Model E 405, Metrohm).

\section{Chemicals and reagents}

All chemicals and standard solutions used were of analytical reagent grade and were purchased from Laboratorijske Kemikalije (Slovenia), Kemika Zagreb (Croatia), and Merck. Stock solutions for the preparation of the supporting electrolytes were made $1 \mathrm{~mol} / \mathrm{L}$ in concentration; standard test solutions used were $0.01 \mathrm{M}$. Diluted solutions $(<0.001 \mathrm{~mol} / \mathrm{L})$ were 
freshly prepared daily. In some cases, the solutions were purged with nitrogen gas (purity 99.99\%) prior to analysis. Doubly deionized water was used throughout the work.

\section{Working electrodes}

Carbon pastes were made by intimate hand-mixing of RW-B spectroscopic graphite powder (Ringsdorff Werke, Germany) with the corresponding liquid binder. Three different carbon pastes were prepared: "Uv-CPE" type, containing Uvasol ${ }^{\circledR}$ paraffin oil (Merck), "SO-CPE" with Lukooil silicone oil (MV 15500, Lučební závody Kolín, Czech Republic), and "TCPCPE" prepared from tricresyl phosphate (mixture of isomers, Fluka). All three pastes homogenized at the same ratio of $0.5 \mathrm{~g}$ graphite $+0.2 \mathrm{~mL}$ liquid were packed into self-designed electrode bodies equipped with a piston for extrusion of the paste. ${ }^{14}$ The electrode surface of CPEs ( $2 \mathrm{~mm}$ in diameter) was renewed mechanically by smoothing some paste off with a wet piece of filter paper.

\section{Procedure}

If not stated otherwise, a typical measurement procedure started with mechanical electrode surface renewal followed by its conditioning and the deposition at potentials and for a time period chosen depending on the model analyte type. After equilibrating, typically for $15 \mathrm{~s}$, the electrode with the deposited analyte was stripped off within the selected stripping limits using either a chemical oxidant (in PSA) or a pre-set constant current (CCSA). Those parameters that are not specified here differed from experiment to experiment and their respective values are declared under Results and Discussion.

Stripping measurements were displayed as "d $t / \mathrm{d} E$ vs. E" curves and stored in a form of "ipw." files. The individual analytical signals were evaluated as peak areas using a manual base-line setting.

\section{Results and Discussion}

\section{Choice of carbon paste electrodes}

Both Uv-CPE and SO-CPE were selected as already classical carbon paste electrodes. ${ }^{6}$ Although physico-chemical characteristics and electrochemical behavior of these CPEs are nearly comparable, there are some differences in their behavior (e.g., nuances in polarizability in some media or different stability in solutions containing organic solvents) due to which both Uv-CPE and SO-CPE have been tested simultaneously. The binder used for the preparation of the SO-CPE was a common type of highly viscous silicone oil (m.w. $15500 \mathrm{~g} / \mathrm{mol}$ ) eventually replaceable by any commercially available product with similar consistency. The TCP-CPE chosen as the third electrode represented the carbon paste containing rather reactive pasting liquid. In contrast to chemically inert paraffin or silicone oil, the molecules of tricresyl phosphate are capable of ion-pairing and extracting some lipophilic substances, which makes the TCP-CPE particularly suitable for their determination. ${ }^{14}$ The carbon-to-pasting liquid ratio was chosen accordingly to previous experience ${ }^{14,15}$ and being the same for all three pastes.

Carbon paste electrodes in stripping potentiometry with electrolytic deposition

CPEs containing inert binders were investigated as supports for a mercury- and gold-film plated in situ. In first case, the $\mathrm{Hg}^{2+}$ ions spiked in the supporting electrolyte served also as chemical oxidant in PSA mode. The stripping of the gold-film

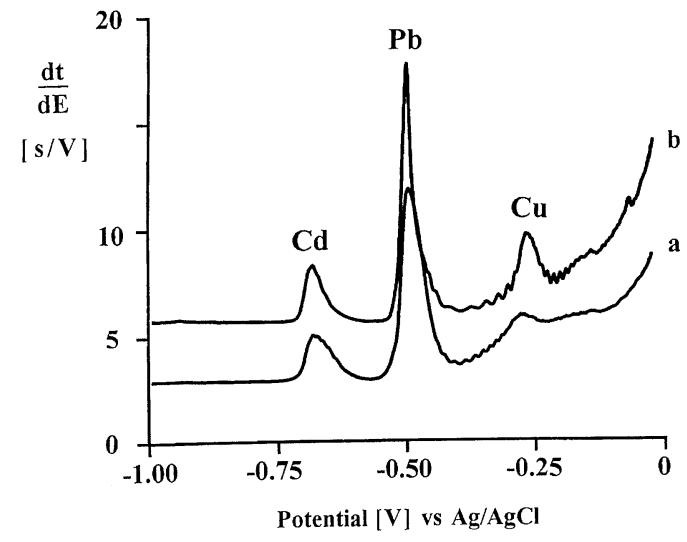

Fig. 1 Potentiometric stripping analysis of $\mathrm{Pb}^{2+}, \mathrm{Cd}^{2+}$, and $\mathrm{Cu}^{2+}$ at CPEs plated with a mercury film. (a) Uv-CPE, (b) SO-CPE. Experimental conditions: model solution, $0.1 \mathrm{M} \mathrm{HCl}+2 \times 10^{-5} \mathrm{M}$ $\mathrm{Hg}^{2+}+1 \times 10^{-6} \mathrm{M} \mathrm{Pb}^{2+}, \mathrm{Cd}^{2+}$ and $\mathrm{Cu}^{2+}$; purging with $\mathrm{N}_{2}$ for $5 \mathrm{~min}$; conditioning potential, $+1.0 \mathrm{~V}$ vs. $\mathrm{Ag} / \mathrm{AgCl}$ (duration, $10 \mathrm{~s}$ ); deposition potential, $-1.0 \mathrm{~V}$; deposition time, $15 \mathrm{~s}$; equilibration time, $10 \mathrm{~s}$; stripping: $-1.0 \rightarrow 0.0 \mathrm{~V}$.

plated CPEs was then performed with constant current. In both cases, the test solutions were purged with nitrogen to remove oxygen as a competitive oxidant of spiked $\mathrm{Hg}^{2+}$ or oxidative current, respectively. ${ }^{1,4}$

The function of mercury film-plated CPEs was observed on the detection of $\mathrm{Pb}^{2+}, \mathrm{Cd}^{2+}$, and $\mathrm{Cu}^{2+}$ at the micromolar concentration level. Figure 1 shows stripping potentiograms obtained by analyzing this model mixture by both Uv-CPE and SO-CPE. The stripping curves manifest well-defined peaks with a satisfactory resolution. It is interesting to notice a substantially higher response towards lead of both mercury-film plated Uv-CPE and SO-CPE compared to those for $\mathrm{Cd}^{2+}$ and $\mathrm{Cu}^{2+}$. This phenomenon is known and, in general, valid in stripping analysis employing mercury film electrodes because lead is reported to form an especially stable amalgam with mercury. ${ }^{3}$ The figure also reveals that the intensity and character of peaks depend also upon the substrate used for plating with a mercury film. This is associated with the capability of the respective substrate to provide optimal surface conditions for the deposition of the film. ${ }^{4}$ Regarding CPEs, the type as well as the quantity of a binder in the carbon paste mixture plays a key-role in the resultant properties of mercuryfilm plated CPEs. ${ }^{16}$

According to previous experience, ${ }^{17}$ the analytical performance of gold film-plated CPEs was tested on the determination of mercury. Figure 2 depicts measurements in CCSA mode with a model concentration of $1 \times 10^{-6} \mathrm{M} \mathrm{Hg}^{2+}$. Even in this assay, the SO-CPE showed somewhat better performance than measurement with the Uv-CPE. Both stripping curves illustrate a wide anodic potential range available for the stripping at the support itself, which is documented by the peak of gold registered still within the operational range. This was exploited for elaborating numerous methods for the determination of gold at CPEs. ${ }^{6,14,18}$

Another experiment served to test the analytical performance of the SO-CPE electrode pre-plated with a gold film from a special plating solution. ${ }^{3}$ The maximum stripping limit was set to $+0.7 \mathrm{~V}$ in order to prevent the oxidative dissolution of the gold film. The carbon paste surface was first renewed mechanically and, after plating with gold, the electrode was tested on six replicate measurements of the above-stated model 


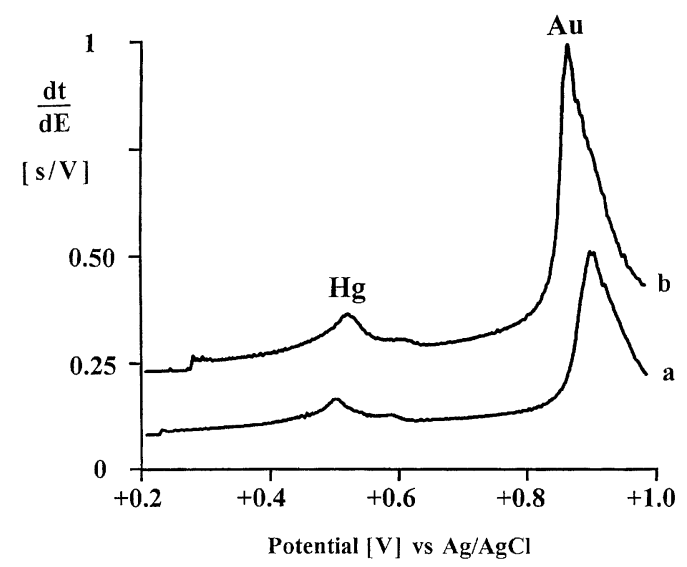

Fig. 2 Constant current stripping analysis of $\mathrm{Hg}^{2+}$ at gold-film plated CPEs. (a) Uv-CPE, (b) SO-CPE. Experimental conditions: model solution, $0.1 \mathrm{M} \mathrm{HNO}_{3}+5 \times 10^{-6} \mathrm{M} \mathrm{Au}^{\mathrm{III}}+1 \times 10^{-6} \mathrm{M} \mathrm{Hg}^{2+}$; purging with $\mathrm{N}_{2}(5 \mathrm{~min})$; conditioning potential, $+1.2 \mathrm{~V}(5 \mathrm{~s})$; deposition potential, $+0.2 \mathrm{~V}$; deposition time, $15 \mathrm{~s}$; equilibration time, $10 \mathrm{~s}$; stripping: $+0.2 \rightarrow+1.0 \mathrm{~V}$; stripping current, $+1 \mu \mathrm{A}$.

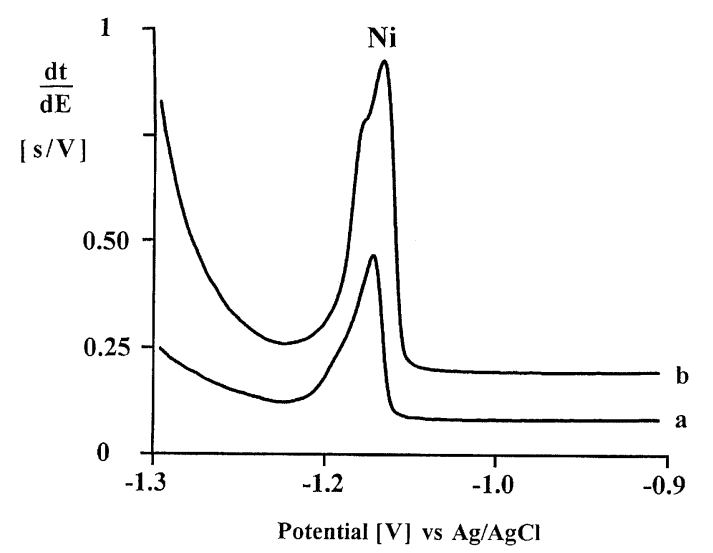

Fig. 3 Constant current stripping analysis of $\mathrm{Ni}^{2+}$ at the SO-CPE modified in situ with dimethylglyoxime. (a) $1 \times 10^{-6} \mathrm{M}$, (b) $2 \times 10^{-6}$ $\mathrm{M} \mathrm{Ni}^{2+}$. Experimental conditions: supporting electrolyte, $0.5 \mathrm{M}$ ammonium buffer $(\mathrm{pH} 9.0)+1 \times 10^{-4} \mathrm{M}$ dimethylglyoxime; purging with $\mathrm{N}_{2}(5 \mathrm{~min})$; conditioning potential, $+0.5 \mathrm{~V}$ (5 s); deposition potential, $-0.2 \mathrm{~V}$; deposition time, $10 \mathrm{~s}$; equilibration time, $15 \mathrm{~s}$; stripping: $-0.5 \rightarrow-1.2 \mathrm{~V}$; stripping current, $-1 \mu \mathrm{A}$.

concentration of $\mathrm{Hg}^{2+}$. The reproducibility calculated as the relative standard deviation was found to be $c a . \pm 3 \%$, which is a value fully comparable to voltammetric experiments. ${ }^{17}$

The voltammograms also reveal some noise superimposed upon the base-line (Fig. 1); eventually, small post-peaks of unspecified origin (Fig. 2). Such signals are sometimes observed in trace electroanalytical measurements; however, they do not usually affect the evaluation of the peak of interest.

\section{Non-electrolytic deposition in stripping potentiometry at carbon} paste electrodes

In this section, experiments were focused on processes utilizing adsorption, extraction and ion-pairing or their combinations. Figure 3 shows the responses obtained by analyzing the test solution with two successive additions of 1 $\mu \mathrm{M} \mathrm{Ni}{ }^{2+}$. The SO-CPE electrode was modified in situ with dimethylglyoxime and used again in CCSA mode. Typically sharp peaks of interest are due to the adsorption of the $\mathrm{Ni}^{\mathrm{II}}$ -

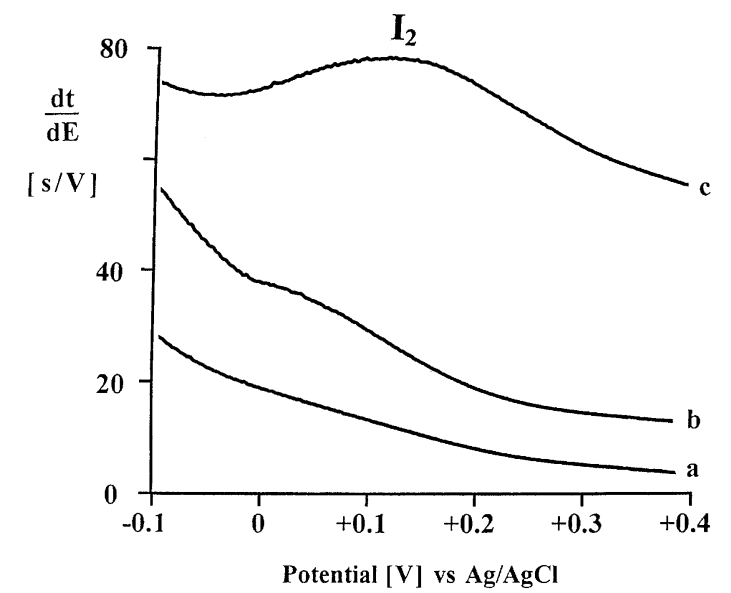

Fig. 4 Determination of iodide in model sample of urine by CCSP with the TCP-CPE. (a) Urine sample, (b) $+0.6 \mathrm{mg} / \mathrm{L} \mathrm{I}^{-}$, (c) $+6 \mathrm{mg} / \mathrm{L}$ $\mathrm{I}^{-}$. Experimental conditions: model sample, $25 \mathrm{~mL}$ urine $+3 \mathrm{~mL}+2$ $\mathrm{M} \mathrm{HCl}+0.5 \mathrm{~mL} 65 \% \mathrm{HNO}_{3}+22 \mathrm{~mL} \mathrm{H} \mathrm{H}_{2} \mathrm{O}$; purging with $\mathrm{N}_{2}(10$ $\mathrm{min})$; conditioning potential, $+0.5 \mathrm{~V}(3 \mathrm{~s})$; deposition potential, +0.7 $\mathrm{V}$; deposition time, $30 \mathrm{~s}$; equilibration time, $15 \mathrm{~s}$; stripping: $+0.7 \rightarrow$ $-0.3 \mathrm{~V}$; stripping current, $-0.5 \mu \mathrm{A}$.

dimethylglyoximate chelate during the deposition step. A small shoulder at the left side of the peaks can be attributed to some desorption effects at the rather heterogeneous surface of the carbon paste. ${ }^{6}$ Such deformations can however be effectively eliminated by optimizing the carbon paste composition or with the aid of appropriate surface pretreatment. ${ }^{10}$

Ion-pair formation combined with extraction was investigated by using the TCP-CPE specially proposed for such a type of synergistic deposition mechanisms. ${ }^{15}$ Since these processes of non-faradayic nature are usually highly selective, the TCP-CPE coupled with CCSA was examined on the determination of iodide. ${ }^{11}$ Such a method, based on accumulation capabilities of protonated tricresyl phosphate, was tested as adapted to urine samples. This can be seen in Fig. 4, depicting the detection of iodide in the presence of a complex matrix of unpretreated urine diluted only with a supporting electrolyte. This figure illustrates clearly that the TCP-CPE in combination with the CCSA is potentially promising for the measurement of iodide in urine as the natural content of iodide in urine usually varies within a very wide range of $100 \mathrm{ng} / \mathrm{L}$ up to $2 \mathrm{mg} / \mathrm{L} .{ }^{19}$ But, this requires adequate optimization measurements resulting in an exact elaboration of a procedure, which is out of the focus of this paper. The curves (a) and (b) also image very broad signals obtained with a CPE via extractive deposition. ${ }^{15}$

\section{Stripping potentiometry with carbon paste electrodes in organic} analysis

Inorganic applications of both PSA and CCSA still significantly prevail over those proposed for analysis of organic substances and biologically important compounds. ${ }^{3,4,9}$ This fact was particularly motivating for some investigations that follow.

Figure 5 makes a comparison between the response for oxidation of L-ascorbic acid at the SO-CPE and the response of the same electrode, but activated according to Baldwin's procedure. $^{20}$ As expected, this electrolytic activation of the electrode surface has led to a marked enhancement of the signal of interest as well as to a desirable shift of this signal. Both these phenomena closely associated with reaction kinetics at CPEs are highly desirable in organic electroanalysis as they allow us to determine substances with totally irreversible 


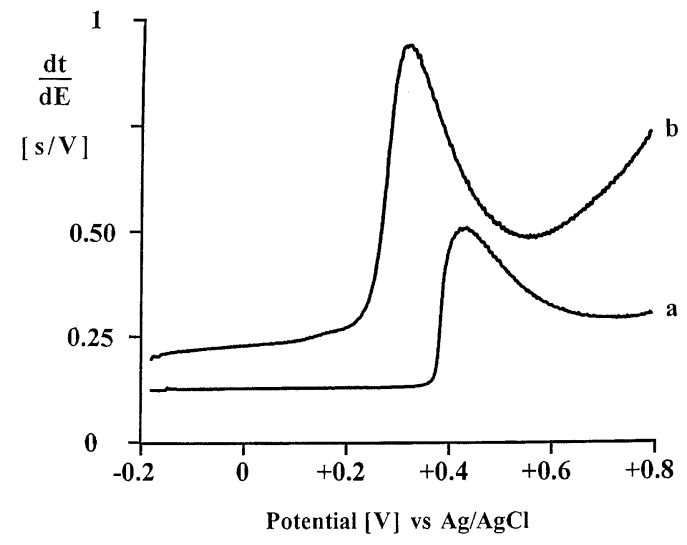

Fig. 5 Oxidation of L-ascorbic acid at the SO-CPE with unactivated and activated surface. (a) Unactivated, (b) activated electrode. Experimental conditions: model solution, $1 \mathrm{M}$ acetate buffer ( $\mathrm{pH} 4.5)$ $+5 \times 10^{-4} \mathrm{M}$ L-ascorbic acid; purging with $\mathrm{N}_{2}(5 \mathrm{~min})$; conditioning potential, $+0.5 \mathrm{~V}(3 \mathrm{~s})$; deposition potential, $-0.5 \mathrm{~V}$; deposition time, $15 \mathrm{~s}$; equilibration time, $10 \mathrm{~s}$; stripping: $-0.2 \rightarrow+0.8 \mathrm{~V}$; stripping current, $+1 \mu \mathrm{A}$; activation procedure prior to CCSA, $+1.75 \mathrm{~V}$ (for 2 $\mathrm{min})$ and $-1.0 \mathrm{~V}(15 \mathrm{~s})$ in $0.1 \mathrm{M}$ phosphate buffer $(\mathrm{pH} 9.1)$.

behavior that cannot normally be detected..$^{20,21}$

Figure 6 depicts a situation in which a CPE has been used in a mixed supporting medium containing $50 \%$ of methanol. Such electrolytes must often be used in order to dissolve numerous organic compounds which are insoluble in pure water solutions. ${ }^{22}$ As the working electrode, the SO-CPE was exclusively selected because of its good stability in mixed media (where other carbon paste mixtures, including the UvCPE type, undergo a rapid disintegration. ${ }^{6,22}$ Both stripping curves in Fig. 6 document that the electrode exhibited a sufficiently stable base-line and was capable of sensitively detecting the anodic oxidation of a model amino compound.

To check the possibilities for bulk modification of CPEs in stripping potentiometry of organic compounds, a model example was examined with the SO-CPE modified with the insoluble salt $\mathrm{PbSO}_{4}$ added directly into the paste mixture (at a ratio of $40 \% \mathrm{w} / \mathrm{w}$ ). This type of CPE modification has been introduced for voltammetric determination of oxalic acid. ${ }^{23}$ Due to the lower solubility of the lead(II) oxalate, the sulfate moiety of the $\mathrm{CPE}$ modifier $\left(\mathrm{PbSO}_{4}\right)$ is replaced by the oxalate anion at the surface of a CPE. The capability of accumulation via this route in combination with CCSA detection of oxalate is displayed in Fig. 7, where a test with unmodified CPE is also shown. Although some further adaptation and optimization experiments are necessary, the difference in performance between the unmodified and modified electrode demonstrates the potential of this approach for the preconcentration and detection of an anionic species.

\section{Conclusions}

Whereas both common CPEs, i.e. Uv-CPE and SO-CPE types, have been shown to have rather universal use, the TCP-CPE can be recommended as an electrode convenient for some special applications. The results and observations have revealed that CPEs represent either a possible alternative to common solid electrodes used in stripping potentiometric measurements or a group of sensors that may potentially extend the existing field of applications of both PSA and CCSA.

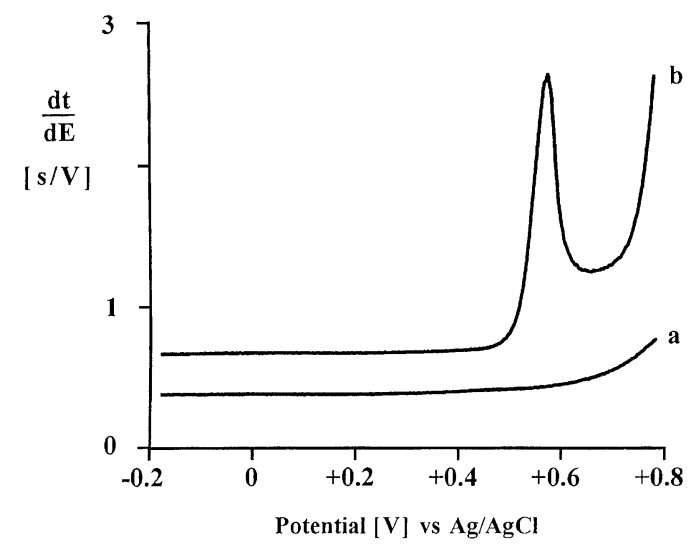

Fig. 6 Oxidation of 3,3'-diammino benzidine at the SO-CPE in methanolic solution. (a) Baseline (supporting electrolyte), (b) 0.001 M 3,3'-diammino benzidine. Experimental conditions: supporting electrolyte, $1 \mathrm{M} \mathrm{H}_{2} \mathrm{SO}_{4}$ in $30 \% \mathrm{CH}_{3} \mathrm{OH}$; purging with $\mathrm{N}_{2}$ (5 min); conditioning potential, $+0.5 \mathrm{~V}(3 \mathrm{~s})$; deposition potential, $-0.2 \mathrm{~V}$; deposition time, $15 \mathrm{~s}$; equilibration time, $10 \mathrm{~s}$; stripping: $-0.2 \rightarrow+0.8$ $\mathrm{V}$; stripping current, $+0.1 \mu \mathrm{A}$.

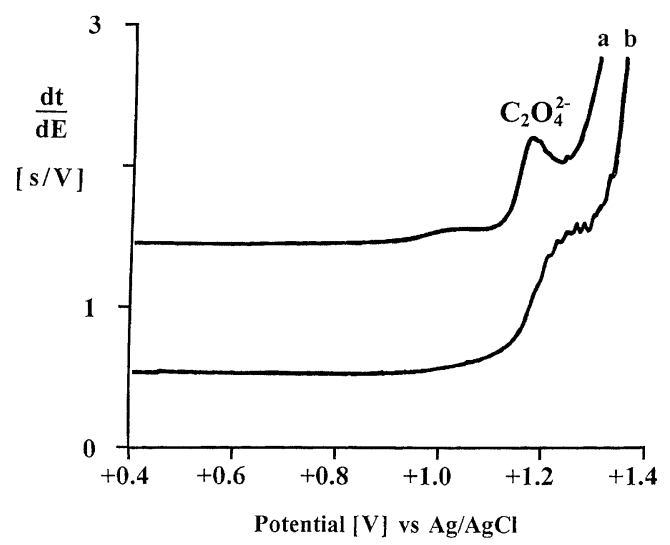

Fig. 7 Determination of oxalic acid at modified and unmodified SO-CPE. (a) Unmodified electrode, (b) electrode modified with $\mathrm{PbSO}_{4} \quad(0.150 \quad \mathrm{~g}: 0.060$ g). Experimental conditions: $0.05 \mathrm{M}$ Britton-Robinson buffer $(\mathrm{pH} 8.0)+1 \times 10^{-5} \mathrm{M}$ oxalic acid; purging with $\mathrm{N}_{2}(5 \mathrm{~min})$; conditioning potential, $+0.5 \mathrm{~V}(3 \mathrm{~s})$; deposition potential, $+0.1 \mathrm{~V}$; deposition time, $30 \mathrm{~s}$; equilibration time, $15 \mathrm{~s}$; stripping: $+0.1 \rightarrow+1.4 \mathrm{~V}$; stripping current, $+1 \mu \mathrm{A}$.

The first is the case of mercury- and gold-film plated carbon pastes that may occasionally replace commonly used glassy carbon or solid gold substrates, belonging among the most frequently used electrodes in both PSA and CCSA. ${ }^{1-5}$ The testing of carbon paste-based substrates for mercury and gold film electrodes is of continuing interest. Recent results ${ }^{16,24}$ have indicated that the properties of CPEs important for deposition of metallic films are almost comparable to either glassy carbon or solid gold electrodes and, in some aspects such as regeneration or pretreatment, the carbon paste can be even more advantageous.

Quite promising seems to be the combination of CPEs and stripping potentiometry for some special measurements based on non-electrolytic processes. In particular, highly selective extraction and ion-pairing, both easily feasible with the TCP$\mathrm{CPE}$, offer one various attractive applications, e.g. analyses of samples with very complex matrixes. One of such applications has already been proposed (determination of iodine in table 
salts $^{25}$ ) and it is believed that similar examples will appear soon. For this, however, we should to continue in the development and optimization of the individual methods, including those reported herein as hitherto preliminary assays.

\section{Acknowledgements}

This work was financially supported by both the Ministry of Science and Technology of the Republic of Slovenia (Project No. P1-0509-0104/99) and the Ministry of Education, Youth and Sports of the Czech Republic (Project No. 253100002).

\section{References}

1. D. Jagner, Analyst, 1982, 107, 593.

2. S. Bruckeinstein and J. W. Bixler, Anal. Chem., 1965, 37, 786.

3. P. Ostapczuk, Anal. Chim. Acta, 1993, 273, 35.

4. J. M. Estela, C. Tomás, A. Cladera, and V. Cerdà, Crit. Rev. Anal. Chem., 1995, 25, 91.

5. J. Konvalina and K. Vytřas, Chem. Listy, 2001, 95, 344.

6. K. Kalcher, J.-M. Kauffmann, I. Švancara, K. Vytras, C. Neuhold, and Z.-P. Yang, Electroanalysis, 1995, 7, 5.

7. K. Kalcher, K. Schachl, I. Švancara, K. Vytřas, and H. Alemu, Sci. Pap. Univ. Pardubice, Ser. A, 1997, 3, 57.

8. M. Trojanowicz and W. Matuszewski, Talanta, 1989, 36, 689.

9. J. Wang, G. Rivas, X.-H. Cai, M. Chicharro, N. Dontha, D.-B. Luo, E. Paleček, and P. E. Nielsen, Electroanalysis, 1997, 9, 120.

10. I. Švancara and K. Schachl, Chem. Listy, 1999, 93, 490.

11. K. Vytřas and J. Konvalina, Electroanalysis, 1998, 10, 787.
12. J. Konvalina and K. Vytřas, Chem. Listy, 2001, 95, 505.

13. K. Vytřas, K. Kalcher, I. Švancara, K. Schachl, E. Khaled, J. Ježková, J. Konvalina, and R. Metelka, Electrochem. Soc. Proc. (Chemical and Biological Sensors and Analytical Methods II), ed. M. Butler, P. Vanýsek, and N. Yamazoe, 2001, 18, 277.

14. I. Švancara and K. Vytr̆as, Anal. Chim. Acta, 1993, 273, 195.

15. I. Švancara, J. Konvalina, K. Schachl, K. Kalcher, and K. Vytřas, Electroanalysis, 1998, 10, 435.

16. J. Konvalina, E. Khaled, and K. Vytras, Collect. Czech. Chem. Commun., 2000, 65, 1047.

17. I. Švancara, M. Matoušek, E. Sikora, K. Schachl, K. Kalcher, and K. Vytřas, Electroanalysis, 1996, 8, 827.

18. K. Vytr̆as, I. S̆vancara, F. Renger, M. Srey, R. Van̆ková, and M. Hvízdalová, Collect. Czech. Chem. Commun., 1993 58, 2039.

19. M. Dermelj, Z. S̆lejkovec, A. R. Byrne, P. Stegnar, S. Hojker, M. Porenta, and G. Šestakov, Analyst, 1992, 117, 443.

20. K. Ravinchandran and R. P. Baldwin, Anal. Chem., 1984, $56,1744$.

21. M. Rice, Z. Galus, and R. N. Adams, J. Electroanal. Chem., 1983, 143, 89.

22. I. Švancara, K. Vytr̆as, F. Renger, and M. R. Smyth, Electrochim. Acta, 1992, 37, 1355.

23. X.-H. Cai, B. Ogorevc, G. Tavčar, and K. Kalcher, Electroanalysis, 1995, 7, 639.

24. E. Khaled, R. Metelka, I. Švancara, K. Vytřas, and H. N. A. Hassan, Abstracts of the 8th International Conference on Electroanalysis, ESEAC 2000, Bonn, Germany, 2000, D26.

25. I. Švancara, B. Ogorevc, M. Nović, and K. Vytřas, J. Anal. Bioanal. Chem., in press. 